\title{
Application of the Beam Theory to Model the Pseudoelastic and Ferroelastic Bending of SMA Beams
}

\author{
L. Orgeas and D. Favier*
}

Laboratoire Génie Physique et Mécanique des Matériaux (GPM2), URA 793 du CNRS, Ecole Nationale Supérieure de Physique de Grenoble, BP. 46, 38042 Saint-Martin-d'Hères cedex, France

* Laboratoire Sols Solides Structures (3S), URA 1511 du CNRS, Université Joseph Fourier, BP. 53X, 38041 Grenoble cedex, France

\begin{abstract}
The study of simple beams made of shape memory alloys (SMA) is very interesting for industrial and experimental reasons. In this paper, it is realised using strength of materials' approach: different kinematical and behavioural assumptions are successively used to solve circular and three supports bendings' problems. The kinematical ones consist in taking into account small or large displacements, the friction being or not neglected, whereas the behavioural ones consider different constitutive equations ie. the elastic-perfectly plastic model and the asymmetric elastohysteretic model. The influences of all these hypothesis are finally illustrated in three supports bending simulations.
\end{abstract}

\section{INTRODUCTION}

Different studies have already been made to model the deformation of statically determined simple beams in pure bending [1] or three supports bending [2], using strength of materials' approach. This research is usefull for two purposes. Firstly the strip shape is very often used in the industrial applications which otherwise involve mostly wires or helical springs. Secondly the bending tests (4 supports, 3 supports or cantilever beams) are very often performed to characterise the mechanical properties of SMA although it is well known that the stress and strain states are not homogeneous in those tests [3]. The comparison of the results obtained in bending and those obtained with homogeneous tensile or shear tests [4] can only be done if assumptions concerning both kinematical aspects of the deformation of the beam and local stress strain relations are simple. The aim of the present paper is to give a general approach of this problem in two simple cases, circular and symmetric 3 supports bendings, using a set of kinematical and behavioural assumptions. The unknowns which are determined are the displacement of the deformed neutral axis, the stress and strain states at any point of the beam. The first part of this document will be devoted to the description of all the employed hypothesis. In the second part, several constitutive equations are presented and then used to model in the third and fourth parts circular and 3 supports bendings. Finally, we present illustrations concerning the influence of the constitutive equations, the importance of assumptions used to take into account large displacements and frictionnal forces which occur at the supports.

\section{WORK ASSUMPTIONS}

\subsection{Beams' concept}

A bar (dimensions 1.b.h) subject to forces or couples that lie in a plane containing the longitudinal axis of the bar is called a beam. When a beam is loaded by forces, internal stresses arise in it. In general, both normal and shearing stresses occur. In order to determine the magnitude of these stresses at any plane 
section of the beam, it is necessary to know the resultant force $\vec{R}_{i}$ and the bending moment $\vec{M}_{i}(G)$ acting at that section. The loaded beam is cut at the curvilign abscissa $s_{G}$ (fig. 1 ), the equations of the static equilibrum is written for the tin of beam $\mathrm{s}<\mathrm{s}_{\mathrm{G}}$ in the $\mathrm{x}, \mathrm{y}, \mathrm{z}$ axis:

$$
\begin{aligned}
& \vec{R}_{i}=N \vec{x}+T_{y} \vec{y}+T_{z} \vec{z}=-\sum \vec{F}_{\text {ext }}(s<s G)-\sum \int_{S} \vec{f}(s<s G) d S \\
& \vec{M}_{i}(G)=M_{t} \vec{x}+M_{f y} \vec{y}+M_{f z} \vec{z}+=-\sum \overrightarrow{C e x t}(s<s G)-\sum \int_{S} \overrightarrow{G M}^{\wedge} \vec{f}(s<s G) d S
\end{aligned}
$$

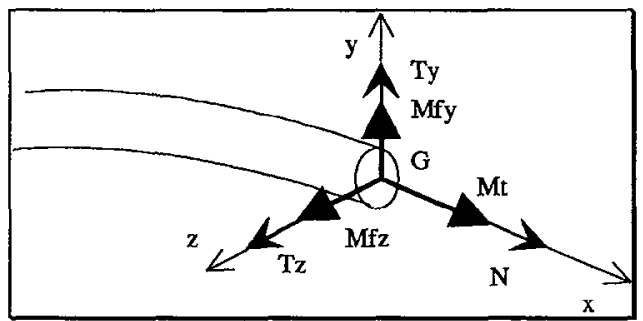

Figure 1: internal forces.

Strictly speaking, these equations are valid only once the beam is deformed. This remark is important for the application of SMA which involve very often large displacements. If $\mathrm{l}>\mathrm{b}$ and $\mathrm{h}$, the bending of the beam can be considered as a two dimensionnal problem, and the shearing stresses can be neglected. The expressions of the internal normal stresses $\sigma_{x x}$, the bending radius $R(x)$ and the distance $d(x)$ between the neutral axis position and the longitudinal axis are given by the following system:

$$
N+\int_{S} \sigma_{x x} d S=0 \quad \text { and } \quad M_{f z}+\int_{S}(y-d) \sigma_{x x} d S=0
$$

\subsection{Navier-Bernouilli's hypothesis, strain and displacement fields}

Navier-Bernouilli's hypothesis consists in supposing that a plane section of the beam normal to its longitudinal axis prior to loading remains plane and normal to the deformed neutral axis after the loading. If small deformation theory is used, the longitudinal deformation $\varepsilon_{x x}$ is simply given by: $\varepsilon_{\mathrm{xx}}=-(\mathrm{y}-\mathrm{d}) / \mathrm{R}$. Supposing that $\mathrm{l} \gg \mathrm{b}$ and $\mathrm{h}$, the deformation of the beam due to transversal strains $\varepsilon_{\mathrm{yy}}$ and $\varepsilon_{\mathrm{zz}}$ are neglected. In this case, the expression of the bending radius in a fixed cartesian frame $(\mathrm{X}, \mathrm{Y})$ is:

$$
R(X)=\frac{\sqrt[3]{\left(1+Y^{\prime 2}\right)}}{Y^{\prime \prime}} \text { or } R(X)=1 / Y^{\prime \prime} \text { in small displacements. }
$$

\subsection{Constitutive equations}

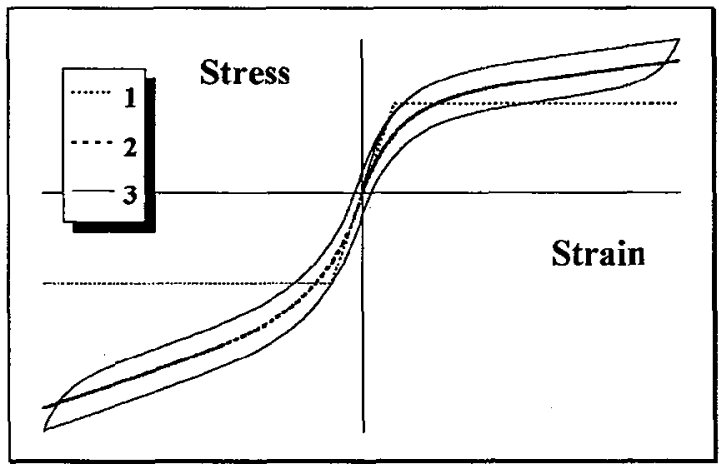

Figure 2: Representation of the three used constitutive equations

\subsubsection{Symmetric elastic-perfectly plastic} constitutive equations

This simple model represented by the curve $n^{\circ} 1$ of fig. 2 needs only two parameters: $E$ and $\sigma_{f}$ which have respectively the meaning of the Young's modulus and the transformation stress.

2.3.2. Non linear reversible behaviour

A more realistic description of the isothermal mechanical behaviour of SMA can be obtained by using a continuous reversible constitutive equations composed of two parts, one in tension and one in compression and defined by 5 parameters $\left(E_{1}, E_{2 t}\right.$, $\mathrm{E}_{2 \mathrm{c}}, \sigma_{\mathrm{t}}$ and $\left.\sigma_{\mathrm{c}}\right)$ :

$$
\begin{gathered}
\sigma_{\text {reviension }}=\sigma_{\mathrm{t}}\left(1-\mathrm{e}^{\left.\frac{-\left(\mathrm{E}_{1}-\mathrm{E}_{2 \mathrm{t}}\right) \varepsilon}{\sigma_{\mathrm{t}}}\right)}+\mathrm{E}_{2 \mathrm{t}} \varepsilon\right. \\
\text { and } \sigma_{\text {revcompression }}=\sigma_{\mathrm{c}}\left(1-\mathrm{e}^{\frac{-\left(\mathrm{E}_{1}-\mathrm{E}_{2 \mathrm{c}}\right) \varepsilon}{\sigma_{\mathrm{c}}}}\right)+\mathrm{E}_{2 \mathrm{c}} \varepsilon
\end{gathered}
$$


Considering the curve $\mathrm{n}^{\circ} 2$ of fig. 2 , it is obvious that the pseudoelastic behaviour can be rather well modelled by these equations if the hysteresis effect is neglected.

\subsubsection{Elastohysteretic constitutive equations}

This last model [5] considers the total stress as the sum of two contributions, of reversible and hysteretic type respectively. The constitutive equations (3) and (4) take into account the difference between tension and compression. $\sigma_{\mathrm{inv}}$ and $\sigma_{\mathrm{inv}}$ are the values of the stress at the inversion points. $w$ is a parameter which is one during the first load and two for the rest. These last constitutive equations which require 7 parameters (the 5 parameters of $\$ 2.3 .2$. and $E_{\text {hys }}, \sigma_{\text {hys }}$ ) can model either the pseudoelastic or the ferroelastic behaviour of SMA depending on the values of the parameters as it can be seen with the curve $n^{\circ} 3$ of fig. 2 .

$$
\begin{aligned}
& \sigma_{\text {tension }}=\sigma_{\text {revtension }}+\sigma_{\text {inv }}+w \sigma_{h y s}\left(1-\mathrm{e}^{\left.\frac{-E_{h y s}\left(\varepsilon-\varepsilon_{\text {inv }}\right)}{\mathrm{w} \sigma_{\text {inv }}}\right)}\right. \\
& \sigma_{\text {compression }}=\sigma_{\text {revcompression }}+\sigma^{\prime} \text { inv }+w \sigma_{h y s}\left(1-e^{\frac{-E_{h y s}\left(\varepsilon-\varepsilon^{\prime} \text { inv }\right)}{w \sigma_{\text {inv }}}}\right)
\end{aligned}
$$

\section{CIRCULAR BENDING}

\subsection{Advantages}

This test consists in applying a couple $\mathrm{M}$ at the extremities of the beam so that the bending moment in the structure is constant and equal to $-\mathrm{M}$ all along the longitudinal axis. The immediate consequence is that the bending radius $\mathrm{R}$ is constant too, and therefore the bended beam is an arc of a circle. The expression of the displacement at the middle of the beam $f$, and at any point $Y(X)$ can be obtained from geometric considerations:

$$
f=-R+\operatorname{sign}(R) \sqrt{R^{2}-\left(\frac{1}{2}\right)^{2}}=Y(1 / 2) \text { and thus } Y(X)=(R+f)-\operatorname{sign}(R) \sqrt{R^{2}-\left(X-\frac{1}{2}\right)^{2}}
$$

The 4 supports bending test can be considered as a circular bending test only for small displacements since the orientations of the reactions of the supports depend on the deformed shape of the beam.

\subsection{Resolution by using the elastic-perfectly plastic equations}

Three cases are to be considered when $|\mathrm{M}|$ increases:

3.2.1. As long as $|\mathrm{M}| \leq \mathrm{M}_{\max \text { elastic }}=\frac{2 \mathrm{I}_{\mathrm{gz}} \sigma_{\mathrm{f}}}{\mathrm{h}}, \quad \mathrm{R}=\frac{-\mathrm{E} \mathrm{Igz}_{\mathrm{gz}}}{\mathrm{M}} \quad$ with $\quad \mathrm{I}_{\mathrm{gz}}=\frac{\mathrm{b} \mathrm{h}^{3}}{12}$.

3.2.2. If $M_{\max e l a x}<|M|<M_{\max }=\frac{\sigma_{f} b}{4}\left(h^{2}-\frac{l^{2} \sigma^{2}}{3 E^{2}}\right) \quad R=-\operatorname{sign}(M) \sqrt{\frac{3 E^{2}}{b \sigma_{f}^{3}}\left(\frac{b^{2} \sigma f}{4}-|M|\right)}$.

3.2.3. If $|M|=M_{\max }|R|$ is equal to $1 / 2$.

\subsection{Resolution by using the reversible or the elastohysteresis equations}

The expression of the displacements is still given by formula (5). To obtain the bending radius and the neutral axis position, the two equations of system (1) have to be solved:

and

$$
\int_{-\operatorname{sign}(M) h / 2}^{0} \sigma_{\text {compression }} d y+\int_{0}^{\operatorname{sign}(M) h / 2} \sigma_{\text {tension }} d y=0
$$

$$
\frac{-|M|}{b}+\int_{0}^{\operatorname{sign}(M) h / 2}(y-d) \sigma_{\text {tension }} d y+\int_{-\operatorname{sign}(M) h / 2}^{0}(y-d) \sigma_{\text {compression }} d y=0
$$


the tension and compression stresses being given by equations (3) and (4). The two expressions (6) and (7) are both function of $\mathrm{R}(\mathrm{X})$ and $\mathrm{d}(\mathrm{X})$. Thus, an iterative process is used to solve the system [6].

\section{SYMMETRIC THREE SUPPORTS BENDING}

The beam is subject to a concentrated force $F$ at $X=1 / 2$ and freely supported at both ends which are distant of 1 .

\subsection{Analytical resolution by using the elastic-perfectly plastic equations}

In such a case, the assumption of small displacements leads to an analytic solution. Because of the symmetry, only half of the beam $(0<\mathrm{X}<\mathrm{l} / 2)$ is considered. The small displacements assumption allows to write the static equilibrum in the initial configuration, so that $\mathrm{Mfz}_{\mathrm{f}}(\mathrm{X})=-\mathrm{F} \mathrm{X} / 2$. During the increase of $\mathrm{F}$, three states are successively met:

4.1.1. First state: $|\mathrm{F}| \leq\left|\mathrm{F}_{\max \text { elas }}\right|=\frac{2 \sigma \mathrm{b} \mathrm{h}^{2}}{3 \mathrm{l}}$ the problem is elastic and: $\mathrm{Y}(\mathrm{X})=\frac{\mathrm{F}}{\mathrm{E} \mathrm{b} \mathrm{h}^{3}}\left(-\mathrm{X}^{3}+\frac{3 \mathrm{l}^{2} \mathrm{X}}{4}\right)$

4.1.2. Second state: $\left|\mathrm{F}_{\max \text { elas }}\right| \leq|\mathrm{F}| \leq\left|\mathrm{F}_{\max }\right|=\frac{\sigma_{\mathrm{f}} \mathrm{b} \mathrm{h}^{2}}{1}$, two cases are to be considered:

. if $0<X<X_{1}=\frac{\sigma f b h^{2}}{|F|}$, the behaviour is elastic and then :

$$
Y_{1}(X)=\frac{-F X^{3}}{E_{b h^{3}}}+K_{1} X=\theta X^{3}+K_{1} X
$$

. if $X_{1}<X<1 / 2$, the system (1) leads to $R(X)=-s \sqrt{\frac{3 E^{2}}{b \sigma_{f}^{3}}\left(\frac{b h^{2} \sigma_{f}}{4}-\left|M f_{z}(X)\right|\right)}$ with $s=\operatorname{sign}(F)$ so that :

$$
\Rightarrow \quad Y_{2}(X)=\frac{4 \alpha}{3 \gamma^{2}} \sqrt[3]{(\beta-\gamma X)}+\frac{2 \alpha}{\gamma} \sqrt{\left(\beta-\gamma \frac{1}{2}\right)} X+C_{1}
$$

with:

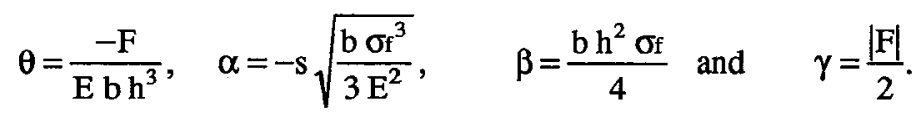

Limit conditions give:

$$
\left\{\begin{array}{l}
\mathrm{K}_{1}=\frac{2 \alpha}{\gamma}\left(\sqrt{\left(\beta-\gamma \frac{1}{2}\right)}-\sqrt{\left(\beta-\gamma \mathrm{X}_{1}\right)}\right)-3 \theta \mathrm{Xl}_{1}^{2} \\
\mathrm{C}_{1}=\theta \mathrm{Xl}^{3}+\mathrm{K}_{1} \mathrm{X}_{1}-\frac{4 \alpha}{3 \gamma^{2}} \sqrt[3]{\left(\beta-\gamma \mathrm{X}_{1}\right)}-\frac{2 \alpha}{\gamma} \sqrt{\left(\beta-\gamma \frac{1}{2}\right)} \mathrm{X}_{1}
\end{array}\right.
$$

4.1.3. Third state: if $|\mathrm{F}| \geq|\mathrm{F} \max |$, plastic localisation is observed at the middle of the beam which is not strong enough to support the bending moment.

\subsection{Resolution by using the reversible or the elastohysteresis equations}

\subsubsection{Kinematical hypothesis}

To determine the solution, small displacements theory is here left out. Thus we must take into account both the current shape of the deformed beam and the variation of reaction's direction (at $X=0)$ during the increase of $\mathrm{F}$ (fig. 3): if the friction is neglected, this force is always normal to the deflection curve of the beam at $X=0$ and its projection on the $Y$ axis is equal to $-F / 2$. Moreover if friction between the beam and its supports is considered and modelled by a Coulomb coefficient $f$, the expression of the bending moment becomes: 


$$
M(X)=\frac{\left.-F \cdot X \cdot \cos \left(\alpha_{0}-\text { sdf. } \varphi\right)-\beta(X)\right)}{2 \cdot \cos \left(\alpha_{0}-\operatorname{sdf} \cdot \varphi\right) \cdot \cos (\beta(X))} \quad \text { with } \beta(X)=\operatorname{Arctan}(Y(X) / X)
$$

and $\quad \operatorname{sdf}=\operatorname{sign}($ incremental load $\mathrm{dF}), \quad \alpha_{\mathrm{o}}=\operatorname{Arctan}\left(\mathrm{Y}^{\prime}(0)\right), \quad \varphi=\operatorname{Arctan} \mathrm{f}$

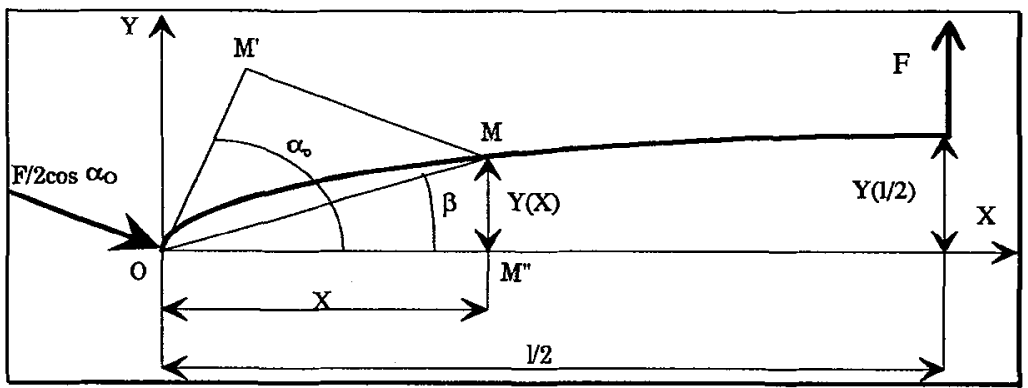

Figure 3: influence of large displacements on the expression of the bending moment.

\subsubsection{Determination of the displacements}

Similarly to 3.3, system (1) is solved iteratively with Mfz given by equation (8), the displacements being obtained by an Euler integration method [6].

\section{MLUSTRATIONS}

The simulations which are here presented concern the symmetric three supports bending test, with a beam of $80 \mathrm{~mm} \times 8.6 \mathrm{~mm} \times 2.7 \mathrm{~mm}$. The following figures show diagramms $F-Y(1 / 2)$.

\subsection{Influence of cinematic hypothesis}

The first illustration (Fig. 4) is obtained adopting the reversible constitutive equations with the following datas: $\mathrm{E}_{1}=90 \mathrm{GPa}, \mathrm{E}_{2 \mathrm{r}}=\mathrm{E}_{2 c}=10 \mathrm{GPa}, \sigma_{t}=\sigma_{c}=500 \mathrm{MPa}$. The curve $\mathrm{n}^{\circ} 1$ is realised with small displacements' assumption, the curve $n^{\circ} 2$ with large displacements, whereas the curve $n^{\circ} 3$ does the same with a friction coefficient $f=0.2$. Comparing curves $n^{\circ} 1$ and $n^{\circ} 2$, it is clear that small displacements' hypothesis tends to "harden" the beam when the deflection increases. The contribution of $f$ can't be neglected too when the displacements become large, as it is shown by comparing the curves 2 and 3: for the maximum plotted force $F$ corresponding to a deflection of the order of $10 \mathrm{~mm}, Y(1 / 2)$ is decreased of 1 $\mathrm{mm}$ by considering friction. This last observation is still supported by the figure 5 where the elastohysteretic theory $\left(\sigma_{\text {hys }}=40 \mathrm{MPa}, \mathrm{E}_{\mathrm{hys}}=50 \mathrm{GPa}\right)$ is used: for a same cyclic load, the beam's answer is completely different with friction (curve $n^{\circ} 1, f=0.2$ ), or without friction (curve $n^{\circ} 2, f=0$ ).

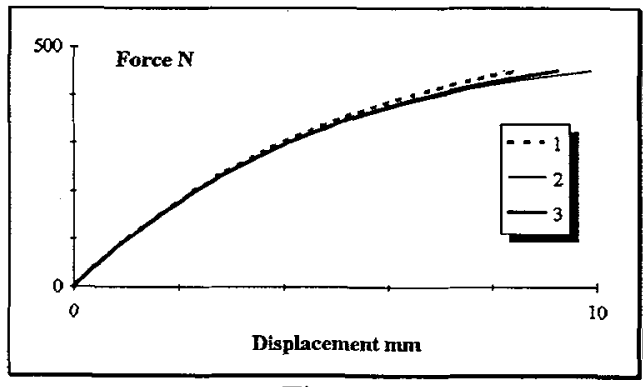

Figure 4

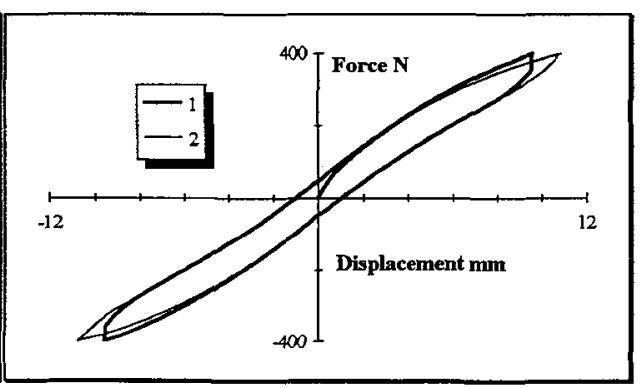

Figure 5

\subsection{Influence of constitutive equations}

The comparison is realised using constitutive equations of $\$ 2.3 .1$ and $\$ 2.3 .2$, with no friction (Fig. 6). The curve $n^{\circ} 1$ uses the elastic-perfectly plastic constitutive equations with $E=90 \mathrm{GPa}, \sigma_{\mathrm{f}}=500 \mathrm{MPa}$ and 
the analytical solution obtained in $\$ 4.1$. The others use the reversible constitutive equations with the following parameters:

\begin{tabular}{|l|l|l|l|}
\hline \multicolumn{1}{|c|}{ Curve $^{\circ} 2$} & \multicolumn{1}{c|}{ Curve $^{\circ} 3$} & \multicolumn{1}{c|}{ Curve $\mathrm{n}^{\circ} 4$} & \multicolumn{1}{c|}{ Curve $\mathrm{n}^{\circ} 5$} \\
\hline $\mathrm{E} 1=90 \mathrm{GPa}$ & $\mathrm{E} 1=90 \mathrm{GPa}$ & $\mathrm{E} 1=90 \mathrm{GPa}$ & $\mathrm{E} 1=90 \mathrm{GPa}$ \\
$\mathrm{E}_{2 \mathrm{c}}=\mathrm{E} 2 \mathrm{a}=0 \mathrm{GPa}$ & $\mathrm{E}_{2 \mathrm{c}}=\mathrm{E} 2 \mathrm{t}=0 \mathrm{GPa}$ & $\mathrm{E}_{2 c}=\mathrm{E} 2 \mathrm{t}=10 \mathrm{GPa}$ & $\mathrm{E}_{2 \mathrm{c}}=\mathrm{E}_{2 \mathrm{t}}=10 \mathrm{GPa}$ \\
$\sigma_{\mathrm{c}}=\sigma_{\mathrm{t}}=500 \mathrm{MPa}$ & $\sigma_{\mathrm{t}}=500 \mathrm{MPa}$ & $\sigma_{\mathrm{c}}=\sigma_{\mathrm{t}}=500 \mathrm{MPa}$ & $\sigma_{\mathrm{t}}=500 \mathrm{MPa}$ \\
& $\sigma_{c}=-700 \mathrm{MPa}$ & & $\sigma_{c}=-700 \mathrm{MPa}$ \\
\hline
\end{tabular}

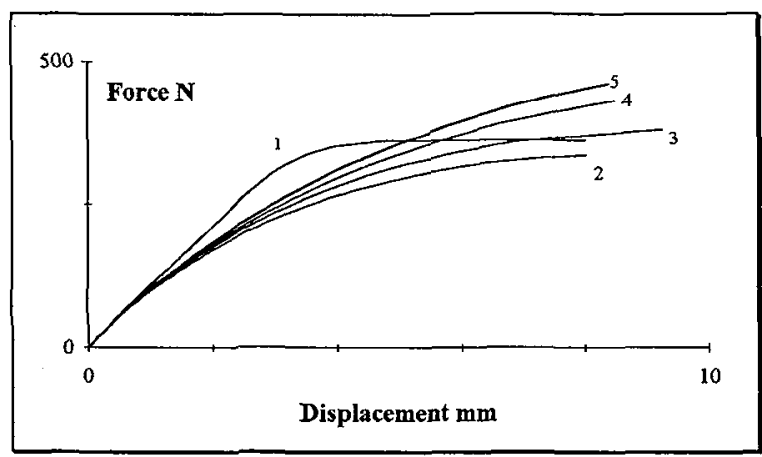

Figure 6
The bending tests have been often used to characterise the mechanical behaviour of SMA [3]. In fact, the analyses of these tests can be performed simply only if ideal symmetric elastic-plastic behaviour and small displacements are considered. In that case, it is possible using analytical resolution of $\$ 4.1$ to get indicative values of the Young's modulus and of an averaged transformation stress $\sigma_{\mathrm{f}}$. However, the experimental stress-strain curves indicate very often no real yield point and a slope associated with the stress induced martensite, even for single cristal [7]. The

consideration of curve 2 shows that the force-displacement curve is greatly affected if the continuous transition between perfect elasticity and perfect plasticity is modelled by a round curve. Moreover, this answer is also modified by the modulus of the stress induced martensite $E_{2}$ (curve 4), by a behavioural asymmetry between compression and tension (curve 3 ) and by the superposition of these two (curve 5).

\section{CONCLUSION}

In this paper, the modelling of bending of SMA has been performed using strength of materials' concept. Adopting restrictive assumptions for stress strain relations and for the extent of displacements, an analytical solution has been established. A general numerical tool has been developped as a software program to get a more realistic solution considering large displacements and friction. The stress-strain relations in tension and compression are symmetric or not, and hysteresis effect can be taken into account, allowing to model both pseudoelastic and ferroelastic behaviours.

\section{References:}

[1] Thiers M., Mick A., Drescher D., Bourauel C., Journal of materials science 26, (1991), 6473-6478.

[2] Gillet Y., Ph. D Thesis, Université de Metz, France, (1994).

[3] Rodriguez P., Guénin G., Materials Science Forum, vol. 56-58, (1990), 541-546.

[4] Manach P.Y., Favier D., "Comparison of homogeneous shear and tensile tests on a NiTi shape memory alloy", Int. Conf. on Mart. Transf., Monterey, USA, (1992), 941-946.

[5] Favier D., Guélin P., Pégon P., Materials Science Forum, vol. 56-58, (1990), 559-564.

[6] Orgeas L., Master's Thesis, Institut National Polytechnique de Grenoble, France, (1994).

[7] Schroeder T.A., Wayman C.M., Acta Metall., Vol 27, (1979), 405-417. 